\title{
Uma Emergência Rinológica: Pilha na Fossa Nasal
}

\section{A Rhinological Emergency: Button Battery in the Nasal Cavity}

Joana Maria Soares Ferreira1*, Cristina Aguiar ${ }^{1}$, Sandra Gerós², António Faria de Almeida ${ }^{3}$, Sandra Alves ${ }^{3}$, Artur Condé ${ }^{4}$

*Autor Correspondente/Corresponding Author: Joana Maria Soares Ferreira [joana_msf@hotmail.com]

\section{RESUMO}

Os corpos estranhos nas fossas nasais são causas frequentes de recurso à urgência, sobretudo em idade pediátrica. Na maioria dos casos são inofensivos, podendo permanecer vários dias sem causar lesões. As pilhas sendo uma exceção, representam uma importante ameaça com necessidade de remoção urgente.

Descreve-se o caso de uma criança com 4 anos de idade, observada no Serviço de Urgência com suspeita de corpo estranho na fossa nasal. Apresentava obstrução nasal e rinorreia unilateral sanguinolenta, com 5 horas de evolução. A radiografia dos ossos próprios nasais identificou um objeto redondo, radiopaco com sinal de duplo halo. Mediante a suspeita de uma pilha na fossa nasal, procedeu-se à sua imediata remoção sob anestesia geral.

Pretendemos alertar para a perigosidade deste tipo de objeto, assim como para a necessidade de uma abordagem diagnóstica e terapêutica céleres, a fim de evitar complicações graves.

PALAVRAS-CHAVE: Cavidade Nasal; Corpos Estranhos; Criança; Fontes de Energia Elétrica; Obstrução Nasal

1. Interna de Formação Específica de Otorrinolaringologia do Centro Hospitalar Vila Nova de Gaia e Espinho, Vila Nova de Gaia, Portugal. 2. Assistente Hospitalar de Otorrinolaringologia do Centro Hospitalar Vila Nova de Gaia e Espinho, Vila Nova de Gaia, Portugal. 3. Assistente Hospitalar Graduado de Otorrinolaringologia do Centro Hospitalar Vila Nova de Gaia e Espinho, Vila Nova de Gaia, Portugal. 4. Diretor de Serviço de Otorrinolaringologia do Centro Hospitalar Vila Nova de Gaia e Espinho, Vila Nova de Gaia, Portugal.

Recebido/Received: 03/11/2021 - Aceite/Accepted: 02/12/2021 - Publicado Online/Published Online: 15/12/2021 - Publicado/Published: 31/12/2021

• Autor (es) (ou seu (s) empregador (es)) e Gazeta Médica 2021. Reutilização permitida de acordo com CC BY-NC. Nenhuma reutilização comercial. ${ }^{\circledR}$ Author(s) (or their employer(s)) and Gazeta Médica 2021. Re-use permitted under CC BY-NC. No commercial re-use. 


\section{ABSTRACT}

Foreign bodies in the nose are frequent causes of emergency care, especially in pediatric patients. In most cases they are harmless and can remain for several days without causing injury. Button batteries being an exception, pose a major threat and need urgent removal.

We describe the case of a 4-year-old child seen in the Emergency Department with a suspected foreign body in the nasal cavity. She presented nasal obstruction and bloody unilateral rhinorrhea, with 5 hours of evolution. Radiography of the nasal bones identified a round, radiopaque object with a double halo sign. Upon suspicion of a button battery in the nose, it was immediately removed under general anesthesia.

We intend to alert to the hazards of this type of object, as well as the need for a quick diagnostic and therapeutic approach, in order to avoid serious complications.

KEYWORDS: Child; Electric Power Supplies; Foreign Bodies; Nasal Cavity; Nasal Obstruction

\section{INTRODUÇÃO}

Os corpos estranhos nas fossas nasais são causas frequentes de recurso à urgência, particularmente na idade pré-escolar. ${ }^{1} \mathrm{Na}$ maioria dos casos tratam-se de pequenos objetos, relativamente inofensivos, existentes no ambiente da criança, como pequenos brinquedos, borracha, papel, alimentos, que podem permanecer vários dias sem causar lesões. ${ }^{1,2} \mathrm{O}$ recurso ao serviço de urgência pode ocorrer de imediato, mediante a suspeita do cuidador, ou mais tardiamente, aquando das manifestações clínicas típicas: rinorreia unilateral de cheiro fétido, com possível epistáxis associada. ${ }^{1,3}$

Nas duas últimas décadas, contudo, devido ao uso crescente de pilhas nos dispositivos eletrónicos e brinquedos, tem-se verificado um aumento de incidência deste tipo de corpo estranho, não só no nariz, mas também noutras áreas anatómicas, como ouvidos e sistema gastrointestinal. ${ }^{4}$ Ao contrário dos outros tipos de corpos estranhos, as pilhas são capazes de originar uma reação eletroquímica em contacto com a mucosa, podendo causar necrose e destruição dos tecidos em poucas horas. ${ }^{5}$

O objetivo deste artigo é alertar para a perigosidade deste tipo de objeto, assim como para a necessidade de uma abordagem diagnóstica e terapêutica célere, a fim de evitar complicações graves e potencialmente fatais.

\section{CASO CLÍNICO}

Doente do sexo feminino com 4 anos de idade, caucasiana, previamente saudável. Recorreu ao Serviço de Urgência de Otorrinolaringologia com suspeita de corpo estranho na fossa nasal esquerda, de natureza desconhecida. A doente apresentava obstrução nasal e rinorreia unilateral esquerda, sanguinolenta, com cerca de 5 horas de evolução. Ao exame objetivo, constatou-se área escurecida na cabeça e porção medial do corneto inferior. Não se visualizava o corpo estranho, contudo palpava-se com cureta de Hallpike um objeto de consistência dura na porção posterior da fossa nasal esquerda.

Foi solicitado uma radiografia dos ossos próprios nasais que revelou um corpo estranho radiopaco, redondo, com sinal de "degrau" em incidência ântero-posterior e sinal de "duplo halo" em incidência de perfil (Fig.s 1 e 2).

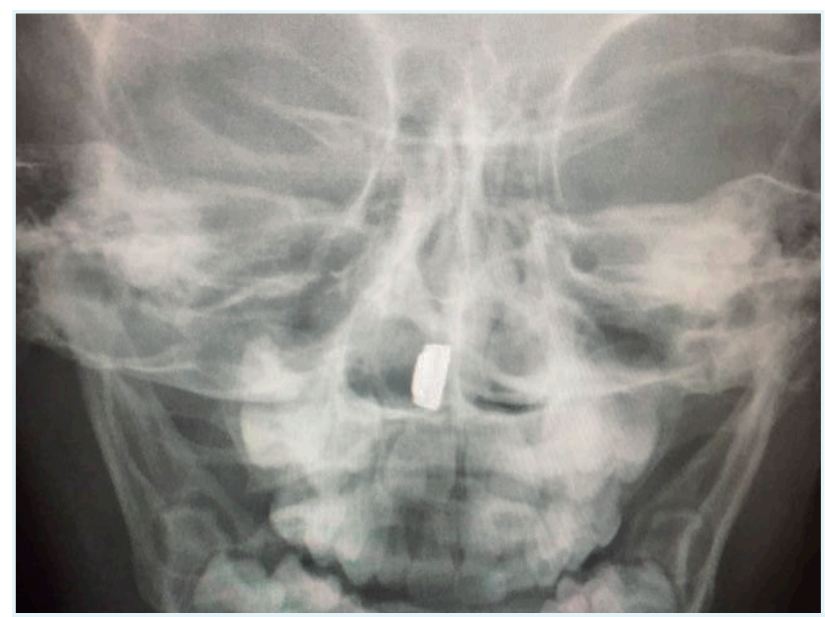

FIGURA 1. Radiografia dos ossos próprios nasais, incidência ântero-posterior, revelando corpo estranho na fossa nasal, redondo, radiopaco e com o sinal de "degrau".

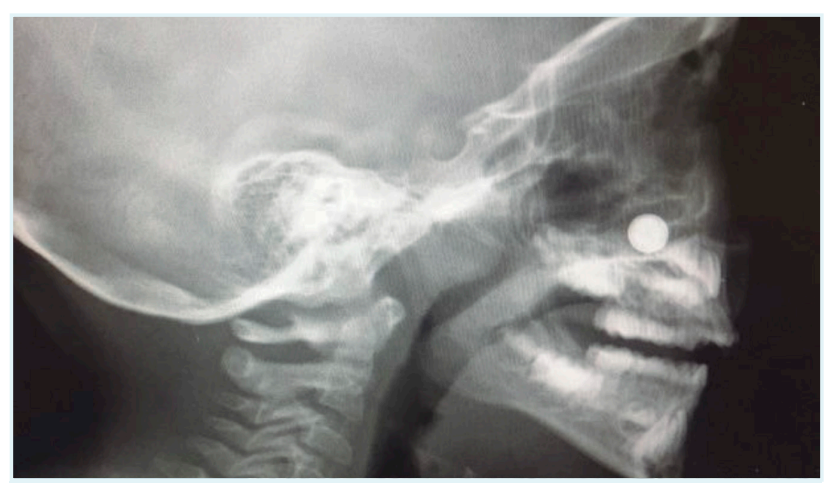

FIGURA 2. Radiografia dos ossos próprios nasais, incidência lateral, revelando corpo estranho no pavimento da cavidade nasal, radiopaco, redondo com o sinal de "duplo halo". 


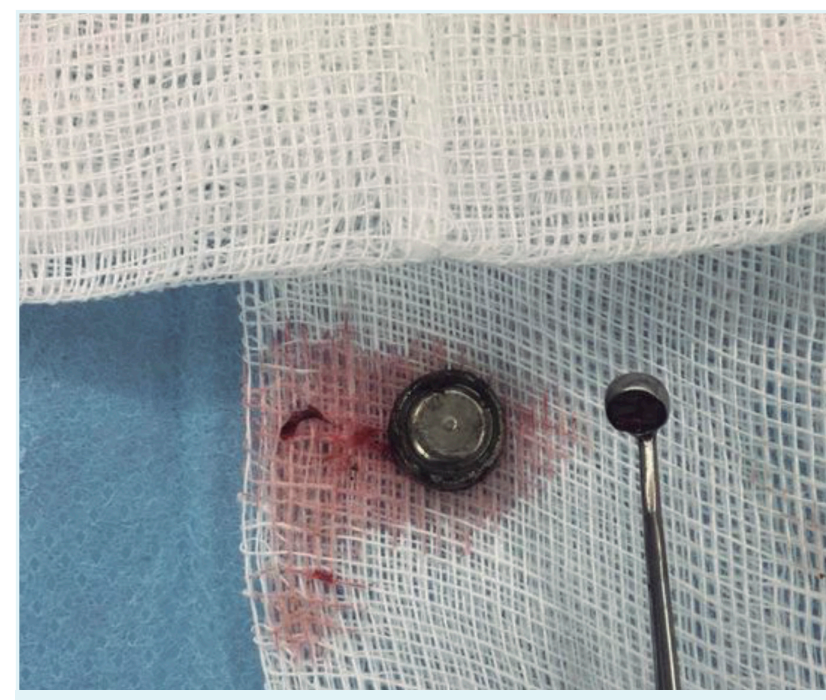

FIGURA 3. Pilha de relógio de óxido de prata removida da fossa nasal com fuga de solução eletrolítica por quebra do selante.

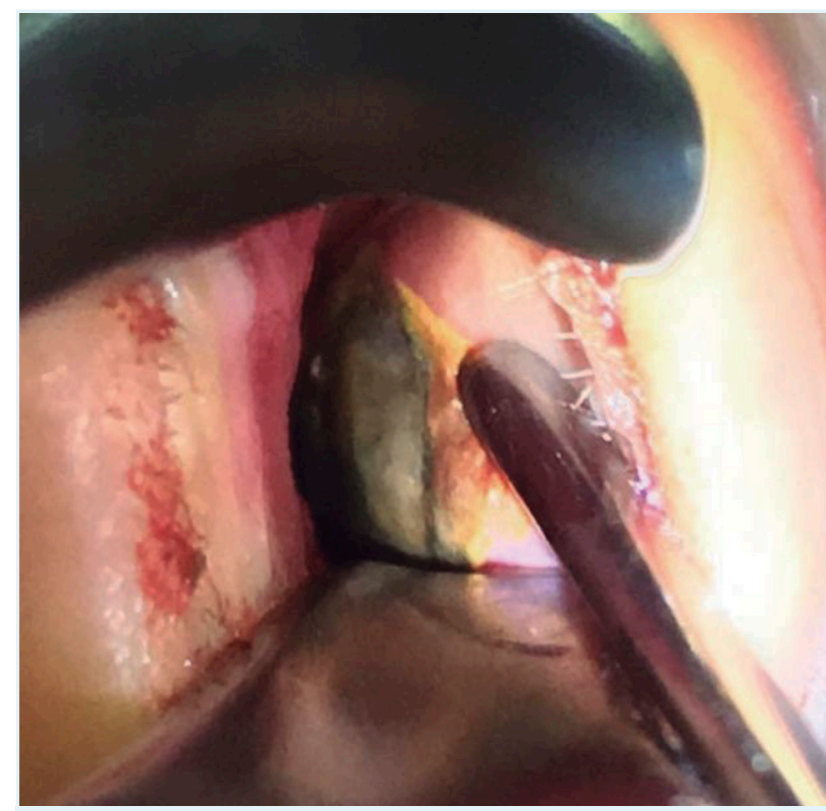

FIGURA 4. Rinoscopia anterior, após remoção de pilha, evidenciando necrose da mucosa do corneto inferior.

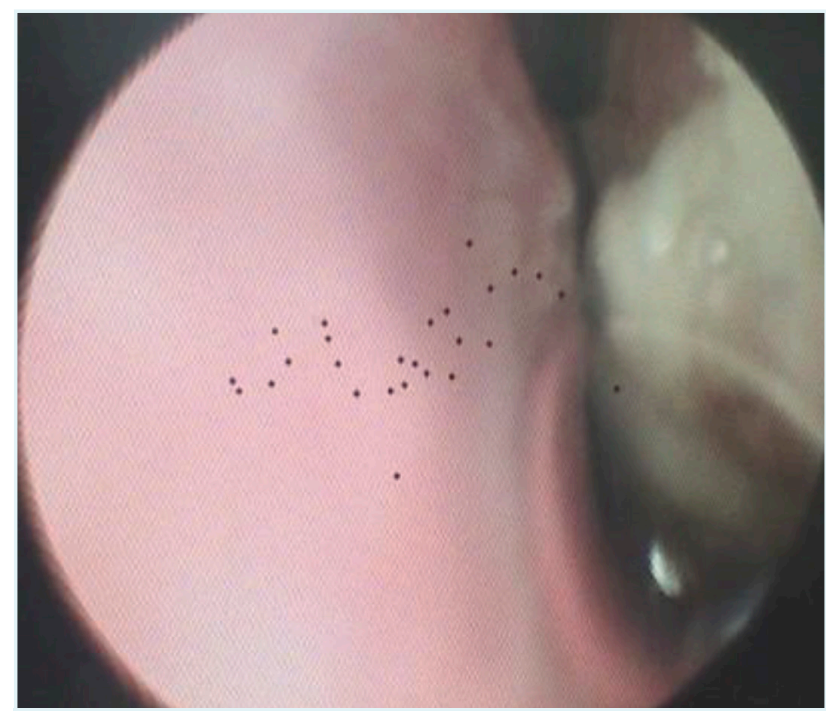

FIGURA 5. Imagem de endoscopia nasal, após 1 mês de seguimento, com área de necrose na cabeça do corneto inferior esquerdo.
Mediante a suspeita de uma pilha na fossa nasal, esta foi prontamente removida sob anestesia geral, confirmando-se tratar-se de uma pilha de relógio de óxido de prata associada a fuga de solução eletrolítica por quebra do selante (Fig. 3). A mucosa do corneto apresentava-se necrosada, com liquefação, e o septo nasal, na área IV de Cottle, revelava um pequeno foco de necrose (Fig. 4). Foi realizado desbridamento das áreas mucosas necrosadas, efetuadas lavagens abundantes de ambas as fossas nasais com água destilada e aplicado antibiótico tópico (bacitracina).

A doente teve alta após 24 horas com recomendações de lavagens nasais frequentes e antibioterapia tópica (bacitracina pomada) e sistémica (amoxicilina com ácido clavulânico).

Após um mês de follow-up, mantinha uma pequena área de necrose na cabeça do corneto inferior, sem outro tipo de complicações, nomeadamente perfuração septal (Fig. 5). Na consulta de reavaliação aos 12 meses, não apresentava qualquer alteração na mucosa nasal.

\section{DISCUSSÃO}

As pilhas na fossa nasal representam uma emergência rinológica, com incidência crescente nas duas últimas décadas. ${ }^{2,3-7}$ Quando em contacto com as superfícies mucosas estes objetos são capazes de provocar lesão através de quatro mecanismos: vazamento do conteúdo eletrolítico da pilha, com alcalinização, liquefação e corrosão dos tecidos; produção de corrente local; dano térmico das estruturas circundantes; e lesão por compressão mecânica.4-6

Várias complicações na fossa nasal estão descritas na literatura, como a necrose e ulcerações das mucosas, celulite orbitária, sinéquias, perfurações septais, desenvolvimento de nariz em sela e até mesmo a morte do doente. ${ }^{3,5,7,8}$

Os principais fatores que determinam o curso clínico, além das características da própria pilha (constituição química, tamanho, voltagem e a carga restante), são o local de impactação e o intervalo de tempo desde a sua introdução até à sua remoção. ${ }^{7,8}$ Sancaktar et al, num estudo de modelo em cadáver, demonstraram que são necessárias apenas duas horas para que uma pilha possa provocar necrose dos tecidos. ${ }^{9}$ Uma série publicada por Loh WS et al revelou que a partir de sete horas de impactação a percentagem de perfurações septais pode ser superior a $70 \% .5$

Dada a agressividade deste tipo de corpo estranho urge a necessidade de uma abordagem diagnóstica e terapêu- 
tica céleres a fim de evitar complicações irreversíveis e potencialmente fatais., ${ }^{2,3}$ Para o diagnóstico é necessário, em primeiro lugar, um alto índice de suspeita, uma vez que a faixa etária mais afetada nem sempre colabora na anamnese, e os incidentes frequentemente ocorrem fora do olhar atento dos cuidadores. ${ }^{4,8}$ É importante pesquisar o tipo de objeto, a duração de evolução do quadro assim como sinais e sintomas associados. ${ }^{8}$ A maioria dos corpos estranhos na fossa nasal demoram dias até ao início das manifestações clínicas típicas: obstrução nasal, dor local, rinorreia unilateral de cheiro fétido com eventual epistáxis associada. As exceções são os objetos pontiagudos que podem causar epistáxis unilateral, por causarem lesão nas mucosas, e as pilhas, pelos mecanismos acima descritos. ${ }^{2} \mathrm{O}$ exame objetivo otorrinolaringológico, nomeadamente a endoscopia nasal, pode ser dificultada nesta faixa etária, em particular, pela difícil colaboração. ${ }^{8}$ No caso de doentes não colaborantes ou mediante a suspeita de um corpo estranho radiopaco, a radiografia dos ossos próprios da face, incidências ântero-posterior e lateral, está indicada. ${ }^{1}$ Trata-se de um exame disponível em quase todos os centros, de baixo custo e elevada acurácia. As pilhas apresentam o sinal característico de duplo halo (dupla-densidade) na incidência de perfil da radiografia, e um degrau de separação entre o ânodo e o cátodo na incidência ântero-posterior (sinal do degrau). ${ }^{7}$

Mediante a suspeita de uma pilha na cavidade nasal, dadas as potenciais complicações associadas tempo dependentes, torna-se emergente a sua remoção. Esta pode ser realizada no gabinete de observação, sob anestesia local. Se a criança não colaborar, existirem riscos significativos, nomeadamente de via aérea, ou se anestesia geral puder ser realizada de forma imediata, esta deve ser a opção, sempre que possível. A remoção da pilha sob anestesia geral permite não só uma melhor caracterização da extensão dos danos como também do seu controlo. ${ }^{2,5}$ Após a sua remoção, está preconizada cobertura antibiótica (tópica e sistémica), lavagens com água destilada (de modo a não perpetuar as potenciais reações eletroquímicas do material liquefeito nas mucosas) e vigilância apertada. 4,6

\section{CONCLUSÃO}

Quando no interior das fossas nasais, as pilhas representam uma ameaça, com elevado risco de rotura e fuga do seu conteúdo e rápida instalação de necrose dos tecidos. O risco de complicações, como as perfurações septais ou nariz em sela, é elevado, sendo essencial para a sua prevenção um alto índice de suspeita, assim como uma rápida abordagem.
Os autores apresentam um caso paradigmático cujo diagnóstico e abordagem precoces permitiram um desfecho clínico favorável.

\section{APRESENTAÇÕES/ PRESENTATIONS}

Trabalho apresentado na forma de poster na "Reunião do Núcleo Sul. Reunião do Interno ORL 2019.”

\section{RESPONSABILIDADES ÉTICAS}

CONFLITOS DE INTERESSE: Os autores declaram a inexistência de conflitos de interesse na realização do presente trabalho.

FONTES DE FINANCIAMENTO: Não existiram fontes externas de financiamento para a realização deste artigo.

CONFIDENCIALIDADE DOS DADOS: Os autores declaram ter seguido os protocolos da sua instituição acerca da publicação dos dados de doentes.

CONSENTIMENTO: Consentimento do doente para publicação obtido.

PROVENIÊNCIA E REVISÃO POR PARES: Não comissionado; revisão externa por pares.

\section{ETHICAL DISCLOSURES}

CONFLICTS OF INTEREST: The authors have no conflicts of interest to declare.

FINANCING SUPPORT: This work has not received any contribution, grant or scholarship.

CONFIDENTIALITY OF DATA: The authors declare that they have followed the protocols of their work center on the publication of data from patients.

PATIENT CONSENT: Consent for publication was obtained.

PROVENANCE AND PEER REVIEW: Not commissioned; externally peer reviewed.

\section{REFERÊNCIAS}

1. OhH, Min HJ, Yang HS, Kim KS. Is radiological evaluation necessary to find out foreing bodies in nasal cavity? J Craniofac Surg. 2016;27:e62-4. doi: 10.1097/SCS.0000000000002165.

2. Santos A, Marinho A, Subtil J, Saraiva J. Pilha nas fossas nasais, uma emergência Otorrinolaringológica. Rev Port SPORL-CCP.2015;53:191-3.

3. Buttazzoni E, Gregori D, Paoli B, Soriani N, Baldas S, Rodriguez H, et al; Susy Safe Working Group. Symptoms associated with button batteries injuries in children: an epidemiological review. Int J Pediatr Otorhinolaryngol. 2015;79:2200-7. doi: 10.1016/j.ijporl.2015.10.003. 
4. Lin VY, Daniel SJ, Papsin BC. Button batteries in the ear, nose and upper aerodigestive tract. Int J Pediatr Otorhinolaryngol. 2004;68:473-9. doi: 10.1016/j.ijporl.2003.10.020.

5. Loh WS, Leong JL, Tan HK. Hazardous foreign bodies: Complications of botton batteries in nose. Ann Otol Rhinol Laryngol. 2003;112:379-83.

6. Sancaktar ME, Bakirtas M. A potential post-removel pH neutralization stategy to mitigate nasal nutton battery injuries. Int J Pediatr Otorhinolaryngol. 2020 Jun;133:110011. doi: 10.1016/j.jporl.2020.110011.

7. Thabet $\mathrm{MH}$, Basha WM, Askar S. Button battery foreign bodies in children: hazards, management, and recommendations. Biomed Res Int. 2013;2013:846091. doi: 10.1155/2013/846091.

8. Ramasamy $\mathrm{V}$, Nadarajah $\mathrm{S}$. The Hazards of impacted alkaline battery in the nose. J Family Med Prim Care. 2018;7:1083-5.

9. Sancaktar M E, Bayraktar C, Bakirtas M. Injury mechanism of button batteries in the nasal cavity and possible mitigation strategies during impaction. Laryngoscope. 2020;130:248793. doi: 10.1002/lary.28913. 